\title{
Abordagem Bayesiana da curva de lactação de cabras Saanen de primeira e segunda ordem de parto
}

\author{
Fabyano Fonseca e Silva ${ }^{(1)}$, Joel Augusto Muniz ${ }^{(1)}$, Luiz Henrique de Aquino ${ }^{(1)}$ e Thelma Sáfadi ${ }^{(1)}$
}

(1)Universidade Federal de Lavras, Dep. de Ciências Exatas, Caixa Postal 37, CEP 37200-000 Lavras, MG. E-mail: fabyanobr@ufla.br, jamuniz@ufla.br, Ihaquino@ufla.br, safadi@ufla.br

\begin{abstract}
Resumo - O objetivo deste trabalho foi utilizar o método Bayesiano no ajuste do modelo de Wood a dados de produção de leite de cabras da raça Saanen. Dois grupos de animais da primeira e segunda lactação foram considerados. Amostras das distribuições marginais a posteriori dos parâmetros do modelo de Wood e das funções de produção derivadas desses parâmetros - pico de produção, tempo do pico de produção, persistência e produção total de leite - foram obtidas pelo algoritmo Gibbs Sampler. As inferências foram feitas em cada população e os resultados mostraram diferenças na taxa de decréscimo da produção após o pico e na persistência, indicando maior produção nos animais de segunda lactação. Realizou-se um estudo de simulação de dados para avaliar o método Bayesiano sob diferentes estruturas de matrizes de covariâncias dos parâmetros. Os resultados desse estudo indicam que o método é eficiente no estudo das curvas de lactação quando a matriz de covariância apresenta alta correlação dos parâmetros.
\end{abstract}

Termos para indexação: Gibbs Sampler, matriz de covariância, produção de leite.

\section{Bayesian approach in the lactation curve of Saanen goats from first and second calving orders}

\begin{abstract}
The objective of this work was to use the Bayesian method in the fitting of the Wood's model for milk production of Saanen goats. Two groups of animals from first and second lactation were considered in the analysis. The posterior marginal distributions for each parameter and production functions, peak milk yield, time of peak yield, persistency and total milk production, were obtained via Gibbs Sampler algorithm. The inference was done for each population. The results showed differences in the slope of the curve after the peak and in persistency, indicating highest production for the second lactation. The data were simulated for evaluating Bayesian method under several covariance matrices structures. The simulation results indicate the efficiency of this method for lactation curves studies when the covariance matrices show high correlation for parameters.
\end{abstract}

Index terms: Gibbs Sampler, covariance matrix, dairy production.

\section{Introdução}

O rígido controle da produção é um fator determinante para o sucesso da exploração leiteira e uma forma prática e consistente de obter este controle é pelo estudo da curva de lactação dos animais, a qual pode ser definida como a representação da produção de leite de um animal no decorrer do tempo em cada lactação (Cobuci et al., 2001).

O conhecimento das curvas de lactação de um rebanho auxilia na adequação de técnicas de alimentação e manejo, e no descarte e seleção de animais de acordo com um padrão desejável, preestabelecido de acordo com a capacidade de produção (Gonçalves, 1994). Dessa maneira, a comparação da forma da curva entre grupos distintos de animais, com diferentes composições raciais, idades ao parto, rebanhos e outros tratamentos de interesse, seria de grande importância, pois, mediante essas comparações, poderiam ser obtidas informações sobre a eficiência desses grupos, propiciando um melhor controle de produção (Groenewald \& Viljoen, 2003).

Modelos estatísticos não-lineares são normalmente utilizados na descrição de curvas de lactação, quando ajustados a dados decorrentes de produção de leite em intervalos consecutivos de tempo. Estes modelos geralmente apresentam parâmetros relacionados com taxas crescentes e decrescentes, as quais lhes conferem a não linearidade paramétrica. Por meio de funções desses parâmetros, outras características da curva de 
lactação podem ser obtidas, como a produção de leite no pico e o tempo de permanência, a produção total de leite e a persistência da lactação (Groenewald et al., 1996).

O método freqüentista (quadrados mínimos) de ajustar modelos não-lineares é baseado em processos iterativos, ou, alternativamente, utilizam-se meios visando a linearizar o modelo mediante transformação logarítmica. Porém, em muitos casos, devido às irregularidades nos dados longitudinais e às correlações existentes entre os parâmetros do modelo (Gallant, 1987), esses métodos produzem estimativas irreais, levando à confecção de curvas de lactação atípicas (Lopes, 1994).

Em recentes estudos envolvendo curvas de lactação (Rekaya et al., 2000; Chang et al., 2001; Jamrozik et al., 2001; Groenewald \& Viljoen, 2003), o método Bayesiano foi utilizado com sucesso uma vez que considera todos os parâmetros como variáveis aleatórias, o que reduz, substancialmente, o número de curvas atípicas. Além disso, esse método requer um número menor de dados de produção por animal, pois os conceitos probabilísticos envolvidos diminuem a dependência do ajuste do modelo em relação ao número de dados utilizados (Goodall \& Sprevak, 1985).

O método Bayesiano também permite a obtenção imediata de intervalos de credibilidade, levando em conta a incerteza existente nos parâmetros simultaneamente, sendo, portanto, a estimação por intervalo geralmente mais precisa em relação àquela apresentada pelo método freqüentista (Gelman et al., 1997). Além disso, os resultados deste método são mais informativos quando se trata de comparações de curvas provenientes de tratamentos diferentes, pois na maioria das vezes, a distribuição dos parâmetros de modelos não-lineares, e principalmente de funções obtidas a partir desses parâmetros, não segue uma distribuição normal, o que torna complexo o processo de formulação de testes estatísticos por meio do método freqüientista (Groenewald et al., 1996).

Segundo Box \& Tiao (1973), o método Bayesiano consiste em uma informação a priori, $\mathrm{P}(\theta)$, a respeito dos parâmetros, de uma função de verossimilhança dos dados, $\mathrm{L}\left(\mathrm{y}_{1}, \ldots, \mathrm{y}_{\mathrm{n}} \mid \theta\right)$, e do cálculo da distribuição de probabilidade a posteriori para os parâmetros. Pelo Teorema de Bayes, a distribuição da densidade a posteriori de $\theta, \mathrm{P}\left(\theta \mid \mathrm{S}_{\mathrm{n}}\right)$, é dada por:

$\mathrm{P}\left(\theta \mid \mathrm{S}_{\mathrm{n}}\right)=\frac{\mathrm{L}\left(\mathrm{S}_{\mathrm{n}} \mid \theta\right) \mathrm{P}(\theta)}{\int \mathrm{L}\left(\mathrm{S}_{\mathrm{n}} \mid \theta\right) \mathrm{P}(\theta) \mathrm{d} \theta}$, sendo $S_{n}=\left\{y_{1}, y_{2}, \ldots, y_{n}\right\}$. Como o denominador não depende de $\theta$, tem-se que $\mathrm{P}\left(\theta \mid \mathrm{S}_{\mathrm{n}}\right) \propto \mathrm{L}\left(\theta \mid \mathrm{S}_{\mathrm{n}}\right) \mathrm{P}(\theta)$, ou seja, Posteriori $\propto$ Verossimilhança $\mathrm{x}$ Priori.

Para se inferir com relação a qualquer elemento de $\theta$, a distribuição a posteriori conjunta dos parâmetros, $\mathrm{P}\left(\theta \mid \mathrm{S}_{\mathrm{n}}\right)$, deve ser integrada em relação a todos os outros elementos que a constituem, ou seja, devem ser obtidas as distribuições marginais de cada parâmetro (Rosa, 1998). Esta integração geralmente não é analítica e necessita de algoritmos iterativos especializados, como o Gibbs Sampler (Amostrador de Gibbs), que faz uso da simulação de Monte Carlo e da teoria das Cadeias de Markov (Sorensen, 1996).

O objetivo deste trabalho foi utilizar o método Bayesiano no ajuste do modelo de Wood em dados de produção de leite de cabras da raça Saanen de primeira e segunda lactação.

\section{Material e Métodos}

A produção esperada de leite em $\mathrm{kg} \mathrm{dia}^{-1}$, no tempo $\mathrm{t}$, foi modelada pela função de Wood (Wood, 1967), cuja expressão é a seguinte:

$\mathrm{E}\left[\mathrm{Y}_{\mathrm{ij}}\right]=\mathrm{t}_{\mathrm{ij}} \mathrm{b}_{\mathrm{ij}} \exp \left(\mathrm{a}_{\mathrm{i}}+\mathrm{t}_{\mathrm{ij}}\right)$, $a_{i}>0,0<b_{i}<1$ e $-1<c_{i}<0, i=1,2, \ldots, k$ e $j=1,2, \ldots, n$

em que $Y_{i j}$ representa a produção de leite do animal i no tempo de lactação $t_{i j} ; a_{i}$ corresponde à produção inicial de leite; $b_{i}$ é a taxa crescente de produção até o pico; e $\mathrm{c}_{\mathrm{i}}$ indica a taxa de declínio da produção após o pico.

Supondo que os resíduos do modelo descrito pela equação 1 sejam multiplicativos e independentes, e adotando a transformação logarítmica, este modelo pode ser reescrito da seguinte maneira:

$\mathrm{Z}_{\mathrm{ij}}=\ln \left(\mathrm{Y}_{\mathrm{ij}}\right)=\mathrm{a}_{\mathrm{i}}+\mathrm{b}_{\mathrm{i}} \ln \left(\mathrm{t}_{\mathrm{ij}}\right)+\mathrm{c}_{\mathrm{i}} \mathrm{t}_{\mathrm{ij}}+\varepsilon_{\mathrm{ij}}$, sendo $\varepsilon_{\mathrm{ij}} \mathrm{o}$ erro experimental, normalmente e independentemente distribuído com média 0 e variância $\sigma_{\mathrm{e}}^{2}$.

Outras importantes características econômicas da curva de lactação foram determinadas por funções dos parâmetros a, b e c, sendo elas a produção de leite no pico $(\eta), \eta=(-b / c)^{b} \exp (a-b) ;$ o tempo de ocorrência do pico $(\tau), \tau=\left(-\frac{b}{c}\right)$; a produção total de leite $(\phi), \phi=\sum_{\mathrm{t}=1}^{\mathrm{L}}(\mathrm{t} / \mathrm{w})^{\mathrm{b}} \exp (\mathrm{a}+\mathrm{ct} / \mathrm{w})$, sendo $\mathrm{L}$ a duração da lactação em dias e w o intervalo de tempo entre os controles leiteiros; e a persistência da lactação $(\varphi)$, $\varphi=-(b+1) \ln (-c)$. 
A função de verossimilhança obtida da expressão 2 é dada por:

$L(Z \mid \theta)=\frac{1}{\left(2 \pi \sigma_{e}^{2}\right)^{N / 2}} \exp \left\{-\frac{1}{2 \sigma_{e}^{2}} \sum_{i=1}^{k} \sum_{j=1}^{n}\left[Z_{i j}-a_{i}-b_{i} \ln \left(t_{i j}\right)-c_{i} t_{i j}\right]^{2}\right\}$.

As distribuições a priori utilizadas para os parâmetros $\mathrm{m}_{\mathrm{i}}, \mathrm{m}_{\mathrm{i}}=\left[\mathrm{a}_{\mathrm{i}}, \mathrm{b}_{\mathrm{i}}, \mathrm{c}_{\mathrm{i}}\right] ; \mu, \mu=[\alpha, \beta, \gamma]$, sendo $\alpha, \beta$ e $\gamma$, respectivamente, as médias dos parâmetros $\mathrm{a}_{\mathrm{i}}, \mathrm{b}_{\mathrm{i}}$ e $\mathrm{c}_{\mathrm{i}} ; \Sigma$, a matriz de covariância para os parâmetros $\alpha, \beta$ e $\gamma$; e $\sigma_{\mathrm{e}}^{2}$ são representadas respectivamente pelas expressões 3 a 6 :

$\mathrm{p}\left(\mathrm{m}_{\mathrm{i}} \mid \mu, \Sigma\right)=(2 \pi)^{-\mathrm{k} / 2}|\Sigma|^{-\mathrm{k} / 2} \exp \left\{-\frac{1}{2} \sum_{\mathrm{i}=1}^{\mathrm{k}}\left(\mathrm{m}_{\mathrm{i}}-\mu\right)^{\prime} \Sigma^{-1}\left(\mathrm{~m}_{\mathrm{i}}-\mu\right)\right\} ;$

$\mathrm{p}(\mu) \propto$ constante;

$\mathrm{p}(\Sigma \mid \mathrm{R}, \mathrm{v}) \propto|\Sigma|^{-(\mathrm{v}+3+1) / 2} \exp \left[-\frac{1}{2} \operatorname{tr}\left(\Sigma^{-1} \mathrm{vR}\right)\right]$

$\mathrm{p}\left(\sigma_{\mathrm{e}}^{2}\right) \propto \frac{1}{\sigma_{\mathrm{e}}^{2}} ;$

em que a expressão 3 é uma normal multivariada; a expressão 5 é uma distribuição de Wishart invertida com matriz escala vR; e a expressão 6 é a priori não informativa de Jeffreys (Jeffreys, 1961).

De acordo com as definições apresentadas, obtémse a seguinte distribuição conjunta a posteriori:

$\mathrm{p}\left(\mathrm{m}_{\mathrm{i}}, \mu, \sigma_{\mathrm{e}}^{2}, \Sigma^{-1} \mid \mathrm{Z}_{\mathrm{i}}, \mathrm{H}\right) \propto \mathrm{p}(\mathrm{Z} \mid \theta) \mathrm{p}\left(\mathrm{m}_{\mathrm{i}} \mid \mu, \Sigma\right)$

$\mathrm{p}\left(\mu, \sigma_{\mathrm{e}}^{2}\right) \mathrm{p}(\Sigma \mid \mathrm{R}, \mathrm{v})$.

Descrevendo algebricamente a equação 7 , tem-se:

$\mathrm{p}\left(\mathrm{m}_{\mathrm{i}}, \mu, \sigma_{\mathrm{e}}^{2}, \Sigma^{-1} \mid \mathrm{Z}_{\mathrm{i}}, \mathrm{H}\right) \propto \frac{1}{\sigma_{\mathrm{e}}^{2(\mathrm{~N} / 2)+1}}$

$\exp \left\{-\frac{1}{2 \sigma_{\mathrm{e}}^{2}} \sum_{\mathrm{i}=1}^{\mathrm{k}}\left[\left(\mathrm{Z}_{\mathrm{i}}-\mathrm{Xm}_{\mathrm{i}}\right)^{\prime}\left(\mathrm{Z}_{\mathrm{i}}-\mathrm{Xm}_{\mathrm{i}}\right)\right]\right\}$

$\times|\Sigma|^{-\mathrm{k} / 2} \exp \left\{-\frac{1}{2} \sum_{\mathrm{i}=1}^{\mathrm{k}}\left(\mathrm{m}_{\mathrm{i}}-\mu\right)^{\prime} \Sigma^{-1}\left(\mathrm{~m}_{\mathrm{i}}-\mu\right)\right\}$

$\times|\Sigma|^{-(\mathrm{v}+3+1) / 2} \exp \left[-\frac{1}{2} \operatorname{tr}\left(\Sigma^{-1} \mathrm{vR}\right)\right]$

sendo H os hiperparâmetros v e R.

As distribuições condicionais completas a posteriori, necessárias à implementação do algoritmo Gibbs Sampler, foram obtidas da equação (7), e são apresentadas como segue:

$$
\begin{aligned}
& \mathrm{m}_{\mathrm{i}} \mid \mathrm{Z}, \mu, \Sigma^{-1}, \sigma^{2} \sim \mathrm{N}\left(\mathrm{D}^{-1}\left(\mathrm{X}^{\prime} \mathrm{Z}_{\mathrm{i}} / \sigma^{2}+\Sigma^{-1} \mu\right), \mathrm{D}^{-1}\right) ; \\
& \mu \mid \mathrm{Z}, \mathrm{M}, \Sigma^{-1}, \sigma^{2} \sim \mathrm{N}\left(\overline{\mathrm{m}}, \frac{1}{\mathrm{k}} \Sigma\right) ; \\
& \Sigma^{-1} \mid \mathrm{Z}, \mathrm{M}, \mu, \sigma^{2} \sim \mathrm{W}\left((\mathrm{S}+\rho \mathrm{R})^{-1}, \mathrm{k}+\rho\right) ;
\end{aligned}
$$

$\sigma^{2} \mid \mathrm{Z}, \mathrm{M}, \mu, \Sigma^{-1} \sim \mathrm{IG}\left(\frac{\mathrm{nk}}{2}, \frac{1}{2}\left(\sum_{\mathrm{i}}^{\mathrm{k}}\left(\mathrm{Z}_{\mathrm{i}}-\mathrm{Xm}_{\mathrm{i}}\right)^{\prime}\left(\mathrm{Z}_{\mathrm{i}}-\mathrm{Xm}_{\mathrm{i}}\right)\right)\right)$.

Nas equações 7 a 11:

$\mathrm{Z}=\left(\mathrm{Z}_{1}, \mathrm{Z}_{2}, \ldots, \mathrm{Z}_{\mathrm{k}}\right)^{\prime} ; \mathrm{M}=\left(\mathrm{m}_{1}, \mathrm{~m}_{2}, \ldots, \mathrm{m}_{\mathrm{k}}\right)^{\prime} ; \mathrm{D}=\mathrm{X}^{\prime} \mathrm{X} / \sigma^{2}+\Sigma^{-1}$; $\overline{\mathrm{m}}=\frac{1}{\mathrm{k}} \sum_{\mathrm{i}=1}^{\mathrm{k}} \mathrm{m}_{\mathrm{i}}$ e $\mathrm{S}=\sum_{\mathrm{i}=1}^{\mathrm{k}}\left(\mathrm{m}_{\mathrm{i}}-\mu\right)\left(\mathrm{m}_{\mathrm{i}}-\mu\right)^{\prime}$;

em que N, W e IG representam respectivamente as distribuições Normal Multivariada, Wishart e Gama Inversa.

O algoritmo Gibbs Sampler foi implementado utilizando-se a linguagem IML (Interactive Matrix Language) disponível no software estatístico SAS (SAS Institute, 1990). Considerou-se, em todas as análises efetuadas, um número fixo de 10.000 iterações, com espaçamento entre os pontos amostrados (thin) de 20 observações, e no aquecimento (burn-in) desprezou-se as 4.000 primeiras iterações. As inferências foram realizadas, portanto, considerando as 6.000 iterações restantes.

Amostras das distribuições marginais a posteriori para as funções (pico de lactação, tempo de pico, produção total de leite e persistência da lactação) dos parâmetros do modelo de Wood foram obtidas pela substituição de $\mathrm{a}, \mathrm{b}$ e c pelas suas respectivas estimativas $(\alpha, \beta$ e $\gamma)$ a cada iteração do Gibbs Sampler, obtendo-se assim, ao final do processo, um conjunto de valores representativos dessas distribuições.

Para verificar a convergência do algoritmo Gibbs Sampler, usou-se o critério informal (visualização gráfica da convergência) e o critério de Gelman \& Rubin (1992), os quais foram aplicados pelo pacote computacional BOA (Bayesian Output Analysis), disponível no software $\mathrm{R}$, utilizando os resultados obtidos do programa em linguagem SAS.

Os dados foram obtidos na Fazenda Nevada, no Município de Carmópolis, MG, e constaram de sete controles leiteiros mensais de 16 cabras da raça Saanen de primeira lactação e de 15 dias da segunda lactação, nascidas entre setembro e dezembro de 2001, semiconfinadas e alimentadas com ração balanceada e silagem de milho.

Um sistema de simulação de dados foi estruturado visando, primeiramente, a avaliar os recursos computacionais empregados e também abordar alguns aspectos do método Bayesiano em relação a diferentes estruturas de matrizes de covariâncias assumidas para os parâmetros.

No teste do algoritmo, elaborado em linguagem SAS, foram simulados dados de produção de leite pela opção RANNOR, utilizando-se os seguintes valores 
paramétricos: $\alpha=0,5, \beta=0,5$ e $\gamma=-0,15$. Consideraram-se dez experimentos com 32 animais, por experimento, e cada animal com dez controles leiteiros. Os resultados foram avaliados para verificar se os valores atribuídos aos parâmetros $\alpha, \beta$ e $\gamma$ encontravamse dentro da amplitude do intervalo de credibilidade de 95\% em cada experimento. Nesse processo de análise, o Gibbs Sampler foi implementado com 5.000 iterações e um intervalo entre os valores amostrados de 20 iterações.

Na comparação das estimativas obtidas, em diferentes estruturas de covariância para os parâmetros $\alpha, \beta$ e $\gamma$, consideraram-se as matrizes auto-regressiva de primeira ordem, AR(1), e auto-regressiva heterocedástica de primeira ordem, $\operatorname{ARH}(1)$, cujas formas são dadas, respectivamente, por $\Sigma_{1}$ e $\Sigma_{2}$.

$\Sigma_{1}=\left[\begin{array}{cccc}\sigma^{2} & \rho \sigma^{2} & \ldots & \rho^{\mathrm{p}-1} \sigma^{2} \\ \rho \sigma^{2} & \sigma^{2} & \ldots & \rho^{\mathrm{p}-2} \sigma^{2} \\ \vdots & : & \ldots & : \\ \rho^{\mathrm{p}-1} \sigma^{2} & \rho^{\mathrm{p}-2} \sigma^{2} & \ldots & \sigma^{2}\end{array}\right]$

$\Sigma_{2}=\left[\begin{array}{cccc}\sigma_{1}^{2} & \rho \sigma_{1} \sigma_{2} & \ldots & \rho^{\mathrm{p}-1} \sigma_{1} \sigma_{\mathrm{p}} \\ \rho \sigma_{1} \sigma_{2} & \sigma_{2}^{2} & \ldots & \rho^{\mathrm{p}-2} \sigma_{2} \sigma_{\mathrm{p}} \\ : & : & \ldots & : \\ \rho^{\mathrm{p}-1} \sigma_{1} \sigma_{\mathrm{p}} & \rho^{\mathrm{p}-2} \sigma_{2} \sigma_{\mathrm{p}} & \ldots & \sigma_{\mathrm{p}}^{2}\end{array}\right]$

Na matriz $\mathrm{AR}(1)$ assumiram-se as seguintes situações: $\rho=0,2,0,3,0,5,0,7,0,9$ e $\sigma^{2}=0,05$. Já na matriz ARH(1), considerou-se que $\rho=0,2,0,3,0,5,0,7,0,9$ e $\sigma_{1}^{2}=0,05, \sigma_{2}^{2}=0,03, \sigma_{3}^{2}=0,01$.

As estimativas obtidas a partir desses parâmetros foram comparadas com os valores paramétricos indicados no parágrafo anterior pelo erro quadrático médio $(\mathrm{EQM})$, definido pela fórmula $\mathrm{EQM}=\frac{1}{\mathrm{Q}}\left[\sum_{\mathrm{r}=1}^{\mathrm{Q}}\left(\hat{\theta}_{\mathrm{i}}-\theta_{\mathrm{i}}\right)^{2}\right]$, em que Q é o número de experimentos simulados.

\section{Resultados e Discussão}

As curvas de lactação estimadas nas duas populações não são as curvas médias, mas meramente geradas pelas médias a posteriori das amostras das distribuições marginais dos parâmetros em ambas as populações, ou seja, elas representam as formas relativas do comportamento da produção de leite, em função do tempo, das cabras de primeira e de segunda ordens de parto (Figura 1).

A população de cabras de segunda lactação é mais produtiva, pois apresenta menor taxa de decréscimo da produção pós-pico $(\gamma)$ e maior persistência $(\varphi)$ (Tabela 1). Estes resultados concordam, em parte, com Gonçalves (1996), que aplicou testes de médias após a análise de variância nas estimativas dos parâmetros do modelo de Wood ajustado a dados de cabras Saanen, e obteve diferenças significativas em todos eles, na comparação entre cabras de primeira e segunda lactações. Por outro lado, Gipson \& Grossman (1989) não encontraram efeito significativo de ordem de parto sobre nenhum dos parâmetros do modelo difásico ajustado às lactações de cabras leiteiras.

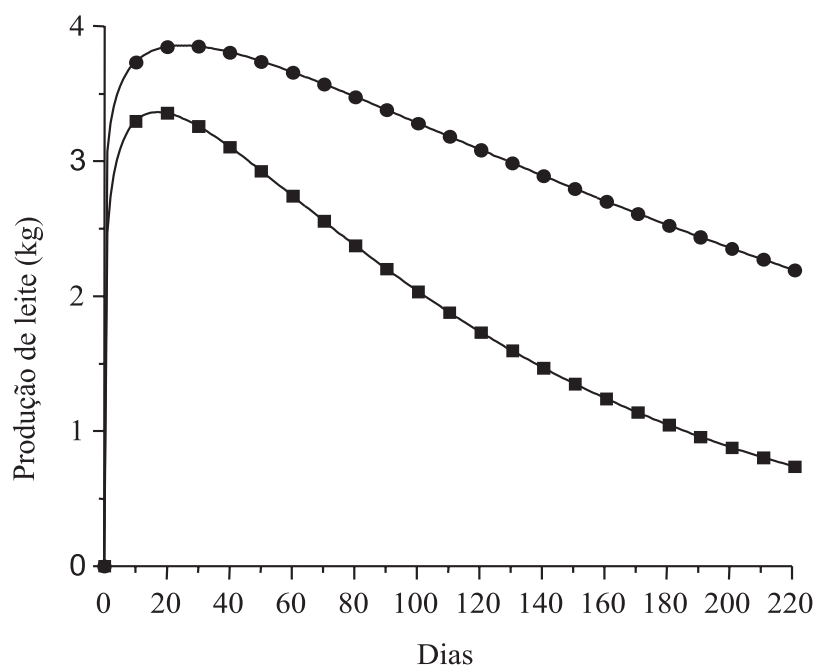

Figura 1. Produção de leite, em função do tempo, pelas cabras de primeira $(\boldsymbol{\square})$ e segunda $(\mathbf{O})$ lactação.

Tabela 1. Estimativas das diferenças entre os parâmetros das duas populações, desvios-padrão e intervalo de credibilidade de $95 \%$.

\begin{tabular}{lrrrr}
\hline Diferença $^{(1)}$ & Média & $\begin{array}{l}\text { Desvio- } \\
\text { padrão }\end{array}$ & $\begin{array}{l}\text { Limite } \\
\text { inferior }\end{array}$ & $\begin{array}{l}\text { Limite } \\
\text { superior }\end{array}$ \\
\hline$\alpha_{1}-\alpha_{2}$ & $-0,2025$ & 0,2852 & $-0,3457$ & 0,7612 \\
$\beta_{1}-\beta_{2}$ & 0,0604 & 0,1065 & $-0,1489$ & 0,2720 \\
$\gamma_{1}-\gamma_{2}$ & $-0,0054$ & 0,0020 & $-0,0094$ & $-0,0015$ \\
$\eta_{1}-\eta_{2}$ & $-0,4418$ & 0,3718 & $-1,1360$ & 0,3101 \\
$\tau_{1}-\tau_{2}$ & $-7,4282$ & 71,5749 & $-31,1545$ & 24,4529 \\
$\phi_{1}-\phi_{2}$ & $-91,7070$ & 74,6205 & $-232,7780$ & 58,9990 \\
$\varphi_{1}-\varphi_{2}$ & $-0,6644$ & 0,2805 & $-1,2134$ & $-0,1256$ \\
\hline
\end{tabular}

${ }^{(1)}$ Subscritos 1 e 2 indicam, respectivamente, primeira e segunda ordem de parto; $\alpha$ é a produção inicial de leite; $\beta$ é a taxa crescente de produção; $\gamma$ é a taxa decrescente de produção; $\eta$ é a produção de leite no pico; $\tau$ é o tempo de ocorrência do pico; $\phi$ é a produção total de leite; $\varphi$ é a persistência da lactação. 
O algoritmo Gibbs Sampler convergiu, em relação a todos os parâmetros, em ambas as populações, pois os valores do coeficiente de convergência (R) estão muito próximos ao valor unitário, o que, segundo Gelman \& Rubin (1992), indica que o processo convergiu (Tabela 2). Além disso, a análise informal da convergência, aspecto visual, mostrou a estabilização do processo (Figura 2) e a forma característica da distribuição marginal (Figura 3), como mostrado para a variância do erro de cabras de primeira lactação. Dessa maneira, podese acreditar que as estimativas dos parâmetros são, verdadeiramente, as médias das distribuições marginais a posteriori, pois a condição de estacionaridade foi atingida.

O método Bayesiano implementado em linguagem SAS mostrou-se eficiente na estimação dos parâmetros $\alpha, \beta$ e $\gamma$, pois os valores paramétricos utilizados na simulação $(\alpha=0,5, \beta=0,5$ e $\gamma=-0,15)$ encontram-se, em todos os experimentos simulados, dentro do intervalo de 95\% de credibilidade (Tabela 3). Resultados semelhantes foram obtidos por Mendoza (1990), que também utilizou este sistema de simulação de dados para avaliar

Tabela 2. Estimativas do coeficiente de convergência $(\hat{R})$ de Gelman e Rubin para os parâmetros das duas populações ${ }^{(1)}$.

\begin{tabular}{cccc}
\hline Parâmetros & $\hat{\mathrm{R}}$ & Parâmetros & $\hat{\mathrm{R}}$ \\
\hline$\alpha_{1}$ & 1,0000470 & $\tau_{1}$ & 1,0026689 \\
$\alpha_{2}$ & 1,0000120 & $\tau_{2}$ & 0,9999110 \\
$\beta_{1}$ & 1,0003553 & $\phi_{1}$ & 1,0005890 \\
$\beta_{2}$ & 1,0002435 & $\phi_{2}$ & 0,9999702 \\
$\gamma_{1}$ & 1,0003925 & $\varphi_{1}$ & 1,0004589 \\
$\gamma_{2}$ & 1,0020534 & $\varphi_{2}$ & 1,0000308 \\
$\eta_{1}$ & 1,0005197 & $\sigma_{1}^{2}$ & 0,9999115 \\
$\eta_{2}$ & 1,0002463 & $\sigma_{2}{ }^{2}$ & 1,0001096 \\
\hline
\end{tabular}

${ }^{(1)}$ Subscritos 1 e 2 indicam, respectivamente, primeira e segunda ordem de parto; $\alpha$ é a produção inicial de leite; $\beta$ é a taxa crescente de produção; $\gamma$ é a taxa decrescente de produção; $\eta$ é a produção de leite no pico; $\tau$ é o tempo de ocorrência do pico; $\phi$ é a produção total de leite; $\varphi$ é a persistência da lactação.

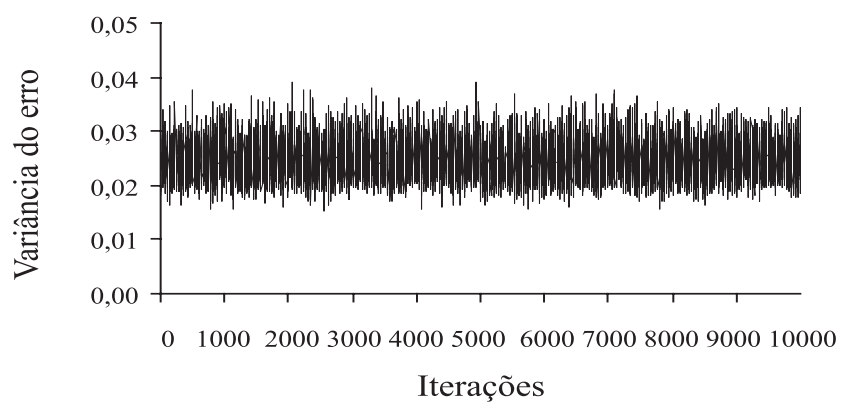

Figura 2. Gibbs Sampler para a variância do erro da população de cabras de primeira lactação.

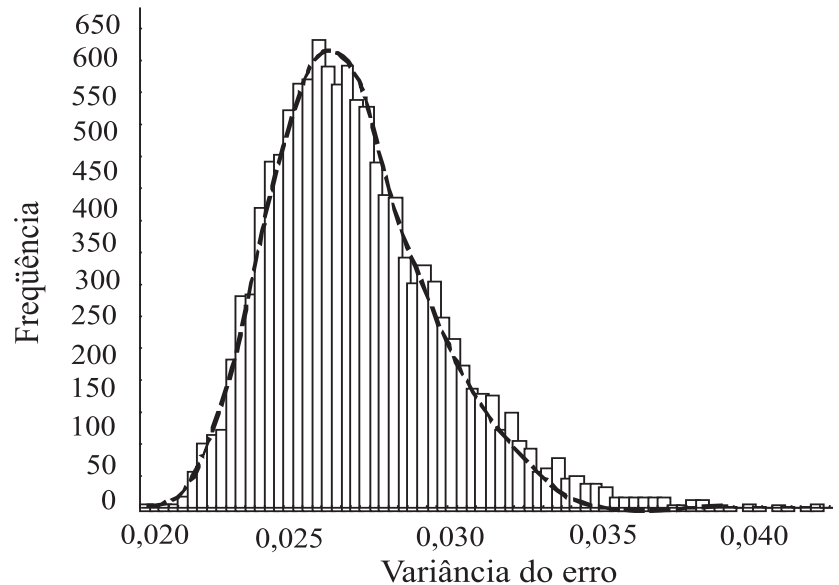

Figura 3. Histograma da amostra da distribuição marginal a posteriori para a variância do erro da população de cabras de primeira lactação.

Tabela 3. Estimativas pontuais e intervalo de credibilidade de 95\% para os parâmetros $\alpha, \beta$ e $\gamma$ nos dez experimentos simulados.

\begin{tabular}{|c|c|c|c|c|}
\hline Experimento & Média & $\begin{array}{l}\text { Desvio- } \\
\text { padrão }\end{array}$ & $\begin{array}{l}\text { Limite } \\
\text { inferior }\end{array}$ & $\begin{array}{c}\text { Limite } \\
\text { superior }\end{array}$ \\
\hline \multicolumn{5}{|c|}{ Parâmetro $\alpha\left(\alpha_{\mathrm{o}}=0,5\right)^{(1)}$} \\
\hline 1 & 0,4594 & 0,1453 & 0,1752 & 0,7459 \\
\hline 2 & 0,4551 & 0,1371 & 0,1868 & 0,7241 \\
\hline 3 & 0,4745 & 0,1414 & 0,1926 & 0,7504 \\
\hline 4 & 0,5267 & 0,1519 & 0,2605 & 0,8779 \\
\hline 5 & 0,5289 & 0,1278 & 0,2796 & 0,7858 \\
\hline 6 & 0,5671 & 0,1578 & 0,2652 & 0,8864 \\
\hline 7 & 0,5295 & 0,1332 & 0,2777 & 0,7926 \\
\hline 8 & 0,4876 & 0,1382 & 0,2257 & 0,7599 \\
\hline 9 & 0,4608 & 0,1589 & 0,1540 & 0,7740 \\
\hline 10 & 0,5252 & 0,1471 & 0,2452 & 0,8212 \\
\hline \multicolumn{5}{|c|}{ Parâmetro $\beta\left(\beta_{\mathrm{o}}=0,5\right)^{(1)}$} \\
\hline 1 & 0,5146 & 0,1781 & 0,1707 & 0,8711 \\
\hline 2 & 0,5475 & 0,1909 & 0,1635 & 0,9213 \\
\hline 3 & 0,5478 & 0,1672 & 0,2152 & 0,8776 \\
\hline 4 & 0,4653 & 0,1935 & 0,0645 & 0,8244 \\
\hline 5 & 0,4823 & 0,1442 & 0,1931 & 0,7611 \\
\hline 6 & 0,3950 & 0,1818 & 0,0329 & 0,7488 \\
\hline 7 & 0,4443 & 0,1790 & 0,0941 & 0,7965 \\
\hline 8 & 0,5455 & 0,1940 & 0,1604 & 0,9060 \\
\hline 9 & 0,5391 & 0,2488 & 0,0563 & 1,0239 \\
\hline 10 & 0,4719 & 0,1516 & 0,1762 & 0,7650 \\
\hline \multicolumn{5}{|c|}{ Parâmetro $\gamma\left(\gamma_{\mathrm{o}}=-0,15\right)^{(1)}$} \\
\hline 1 & $-0,1511$ & 0,0345 & $-0,2241$ & $-0,0881$ \\
\hline 2 & $-0,1562$ & 0,0336 & $-0,2231$ & $-0,0907$ \\
\hline 3 & $-0,1549$ & 0,0288 & $-0,2106$ & $-0,0968$ \\
\hline 4 & $-0,1440$ & 0,0357 & $-0,2144$ & $-0,0746$ \\
\hline 5 & $-0,1479$ & 0,0282 & $-0,2054$ & $-0,0937$ \\
\hline 6 & $-0,1382$ & 0,0339 & $-0,2023$ & $-0,0698$ \\
\hline 7 & $-0,1434$ & 0,0341 & $-0,2108$ & $-0,0786$ \\
\hline 8 & $-0,1601$ & 0,0431 & $-0,2367$ & $-0,0677$ \\
\hline 9 & $-0,1594$ & 0,0418 & $-0,2401$ & $-0,0788$ \\
\hline 10 & $-0,1427$ & 0,0285 & $-0,1974$ & $-0,0872$ \\
\hline
\end{tabular}

(1)Valores paramétricos utilizados na simulação de dados. 
resultados de análises Bayesianas, recomendando sua utilização na avaliação de ensaios de caráter biológico.

À medida que o coeficiente de correlação aumenta, não se verifica uma tendência crescente no valor da raiz quadrada do EQM (Figura 4), que se caracteriza num comportamento típico dos estimadores freqüentistas. Este fato pode estar relacionado com uma menor variabilidade dos estimadores Bayesianos (média da distribuição marginal a posteriori) quando os parâmetros são altamente relacionados. Portanto, estes estimadores podem ser uma alternativa nos estudos de modelos de regressão em que os parâmetros apresentam esta característica.

Não houve grande diferença nos valores da raiz quadrada do EQM, portanto as diferentes variâncias e coeficientes de correlação assumidos em relação aos

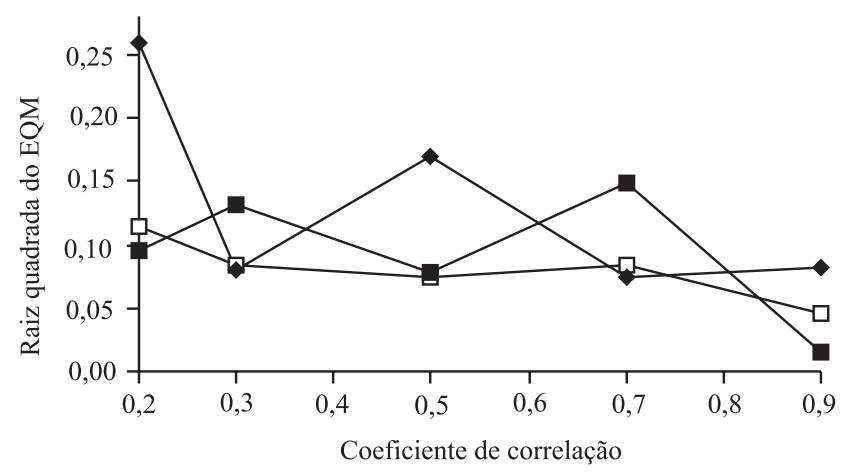

Figura 4. Erro quadrático médio (EQM) em função dos coeficientes de correlação nas estimativas dos parâmetros $\alpha(\square)$, $\beta(\boldsymbol{\square})$ e $\gamma(\vartheta)$, considerando a matriz de covariância autoregressiva de primeira ordem.

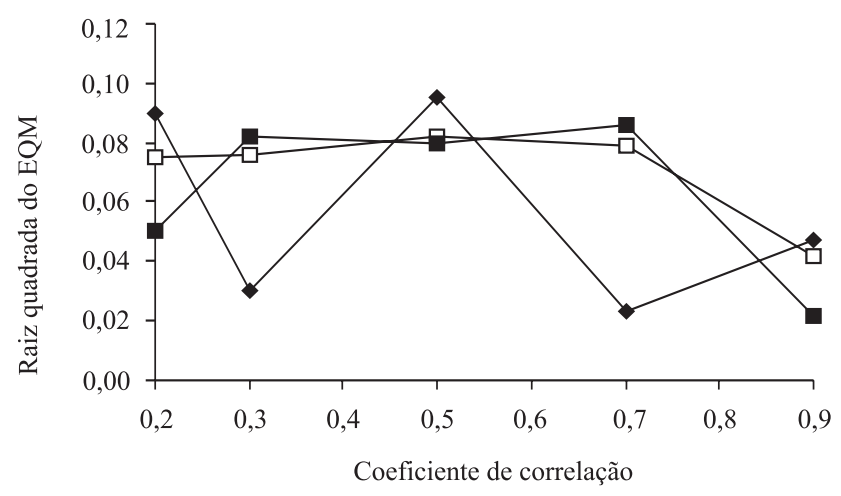

Figura 5. Erro quadrático médio (EQM) em função dos coeficientes de correlação nas estimativas dos parâmetros $\alpha(\square)$, $\beta(\boldsymbol{\square})$ e $\gamma(\downarrow)$, considerando a matriz de covariância autoregressiva heterocedástica de primeira ordem. parâmetros não influenciaram, diretamente, o comportamento do estimador Bayesiano, mostrando-se robusto nas situações de heterogeneidade (Figura 5).

\section{Conclusões}

1. A população de cabras de segunda lactação é mais produtiva que a de primeira lactação.

2. O método Bayesiano é eficiente no estudo das curvas de lactação quando os parâmetros são correlacionados entre si.

\section{Referências}

BOX, G.E.P.; TIAO, G.C. Bayesian inference in statistical analysis. New York: J. Wiley, 1973. 360p.

CHANG, Y.M.; REKAYA, R.; GIANOLA, D.; THOMAS, D.L. Genetic variation of lactation curves in dairy sheep: a Bayesian analysis of Wood's function. Livestock Production Science, v.71, p.241-251, 2001.

COBUCI, J.A.; EUCLYDES, R.F.; TEODORO, R.L.; VERNEQUE, R.S.; LOPES, P.S.; SILVA, M.A. Aspectos genéticos e ambientais da curva de lactação de vacas da raça Guzerá. Revista da Sociedade Brasileira de Zootecnia, v.30, p.1204-1211, 2001.

GALLANT, A.R. Nonlinear statistical models. New York: J. Wiley, 1987. 610p.

GELMAN, A.; CARLIN, J.B.; STERN, H.S.; RUBIN, D.B. Bayesian data analysis. London: Chapman Hall, 1997. 526p.

GELMAN, A.; RUBIN, D.B. Inference from iterative simulation using multiple sequences. Statistical Science, v.7, p.457-511, 1992.

GIPSON, G.S.; GROSSMAN, M. Diphasic analysis of lactation curves in dairy goats. Journal of Dairy Science, v.72, p.10351044, 1989.

GONÇALVES, H.C. Fatores genéticos e de meio em algumas características produtivas e reprodutivas de caprinos. 1996. 141p. Tese (Doutorado) - Universidade Federal de Viçosa, Viçosa.

GONÇALVES, T.M. Estudo da curva de lactação de vacas da raça Gir. 1994. 66p. Dissertação (Doutorado) - Universidade Federal de Viçosa, Viçosa.

GOODALL, A.E.; SPREVAK, D. A Bayesian estimation of lactation curve of dairy cow. Animal Production, v.40, p.189-193, 1985.

GROENEWALD, P.C.N.; FERREIRA, A.V.; MERWE, H.J. van der; SLIPPERS, S.C. Application of Bayesian inference in the comparison of lactation curves of Merino ewes. Animal Science, v.62, p.63-69, 1996.

GROENEWALD, P.C.N.; VILJOEN, C.S. A Bayesian model for analysis of lactation curves of dairy goats. Journal of Agricultural, Biological and Environmental Statistics, v.8, p.75-83, 2003. 
JAMROZIK, J.; GIANOLA, D.; SCHAEFFER, L.R. Bayesian estimation of genetic parameters for test day records in dairy cattle using linear hierarchical models. Livestock Production Science, v.71, p.223-240, 2001.

JEFFREYS, H. Theory of probability. Oxford: Clarendon, 1961. $325 \mathrm{p}$.

LOPES, M.A. Estudo da curva de lactação de um rebanho Holandês variedade preto e branco mantido em um sistema intensivo de produção. 1994. 87p. Dissertação (Mestrado) Universidade Federal de Lavras, Lavras.

MENDOZA, M. A Bayesian analysis of the slope ratio bioassay. Biometrics, v.46, p.1059-1069, 1990.
REKAYA, R.; CARABÃNO, M.J.; TORO, M.A. Bayesian analysis of lactation curves of Holstein-Friesian cattle using a nonlinear model. Journal of Dairy Science, v.83, p.2691-2701, 2000.

ROSA, G.J.M. Análise Bayesiana de modelos lineares mistos robustos via Amostrador de Gibbs. 1998. 57p. Tese (Doutorado) Universidade de São Paulo, Piracicaba.

SAS INSTITUTE (Cary, Estados Unidos). SAS/STAT SAS/IML software: usage and reference: version 6. Cary, 1990. 501p.

SORENSEN, D. Gibbs Sampling in quantitative genetics. Copenhagen: Foulun, 1996. 186p.

WOOD, P.D.P. Algebraic model of the lactation curve in cattle. Nature, v.216, p.164-165, 1967.

Recebido em 11 de novembro de 2003 e aprovado em 8 de setembro de 2004 